\title{
New access to chiral pyrrolidine and piperidine $\beta$-enamino ketones. Application to the enantioselective synthesis of (-)-hygroline
}

\author{
Corinne Vanucci-Bacqué,* Sandrine Calvet-Vitale, Marie-Claude Fargeau-Bellassoued, \\ and Gérard Lhommet* \\ Université Pierre et Marie Curie, Institut de Chimie Moléculaire FR 2769, Laboratoire de \\ Chimie Organique, UMR 7611, Equipe de Chimie des Hétérocycles, 4 place Jussieu, \\ F-75252 Paris cedex 05, France. \\ E-mails:vanucci@ccr.jussieu.fr, lhommet@,ccr.jussieu.fr
}

\begin{abstract}
We report here a new access to chiral pyrrolidine and piperidine $\beta$-enamino ketones by condensation of $(S)$-phenylglycinol with $\omega$-oxo alkynones. As an illustration of the synthetic potential of the target compounds, the total enantioselective synthesis of alkaloid (-)-hygroline was achieved.
\end{abstract}

Keywords: $\beta$-Enamino ketone, pyrrolidine, piperidine, hygroline

\section{Introduction}

Synthesis of $\beta$-enaminones has attracted much interest because of their intrinsic biological properties. ${ }^{1}$ Moreover, $\beta$-enaminones constitute useful precursors for the preparation of a number of heterocycles and natural products. Indeed, due to their versatile reactivity, they can be condensed to fused heterocycles ${ }^{2}$ or be reduced into either $\beta$-amino carbonyl derivatives ${ }^{3}$ or 1,3 amino alcohols. ${ }^{4}$ In our continuing efforts towards the synthesis of natural products, we have been interested in the enantioselective preparation of heterocyclic $\beta$-enaminones bearing an exocyclic double bond. The classical general method for the preparation of such compounds relies on the Eschenmoser sulphide contraction. ${ }^{3 \mathrm{a}, 3 \mathrm{~b},}$ Alternative procedures have been developed to synthesize morpholinone, ${ }^{6}$ pyrrolidine $^{7}$ and piperidine ${ }^{4 a, 7}$ derivatives. More recently, we described the preparation of chiral bicyclic pyrrolidine and piperidine $\beta$-enamino esters $(7 \mathrm{a} R)-\mathbf{1}$ and $(8 \mathrm{a} R)-\mathbf{2}$, by condensation of $(S)$-phenylglycinol with $\omega$-oxo alkynoates $\mathbf{3}$ and $4(\mathrm{R}=\mathrm{OMe})^{8}$ (Scheme 1). During the course of this work, we realized that our strategy could be extended towards the obtention of oxazolidine $\beta$-enamino ketone analogues 5 and $\mathbf{6}$ (Scheme 1). Herein, we wish to report our study concerning the synthesis of these compounds by the condensation of the same chiral amine with various $\omega$-oxo alkynones $\mathbf{3}$ and $\mathbf{4}(\mathrm{R}=$ alkyl, aryl) 
(Scheme 1). The interest of such compounds as precursors of chiral amino alcohols will be demonstrated by the total enantioselective synthesis of the pyrrolidine alkaloid (-)-hygroline.

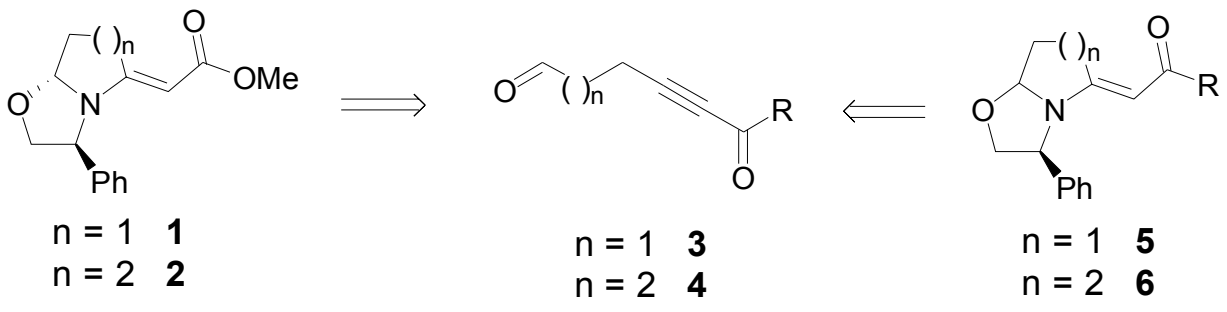

\section{Scheme 1}

\section{Results and Discussion}

To evaluate the feasibility of our approach, we carried out the present study using various alkyl and phenyl alkynones $3(\mathrm{R}=\mathrm{Me}, \mathrm{Ph})$ and $4(\mathrm{R}=\mathrm{Me}, \mathrm{Ph}, n$-Pr, $i$-Pr $)$ as the starting products (Scheme 2). The resulting pyrrolidine and piperidine enamino ketones were viewed as useful building blocks for the total synthesis of various alkaloids. ${ }^{9}$ The required dioxo alkynes $\mathbf{3} \mathbf{a}-\mathbf{b}$ and 4a-d were easily obtained in four steps starting from the tetrahydropyrannyl ether ${ }^{10}$ of pent-4-yn1-ol 7 and hex-5-yn-1-ol 8 (Scheme 2). Condensation of the acetylide anions on the various aldehydes afforded the corresponding propargyl alcohols 9a-b and 10a-d in high yields. Subsequent deprotection of the $\omega$-hydroxy functions was performed using Dowex W50 in methanol to give the corresponding diols $11 \mathbf{a}-\mathbf{b}^{11}$ and 12a-d. During these studies, we noted that the benzylic alcohol (9b and $\mathbf{1 0 b}$ ) were prone to solvolysis by methanol. ${ }^{12}$ Indeed, we observed the formation of the corresponding methyl ethers after prolonged reaction times. ${ }^{12}$ Consequently, the reaction was monitored by gas chromatography and stopped before the appearance of these by-products, even if some unreacted starting material remained. The latter was however easily recovered after column chromatography. Finally, double oxidation of the previously obtained diols was efficiently achieved using Swern conditions ${ }^{13}$ to yield the expected ketoaldehydes 3a-b and 4a-d (Scheme 2).
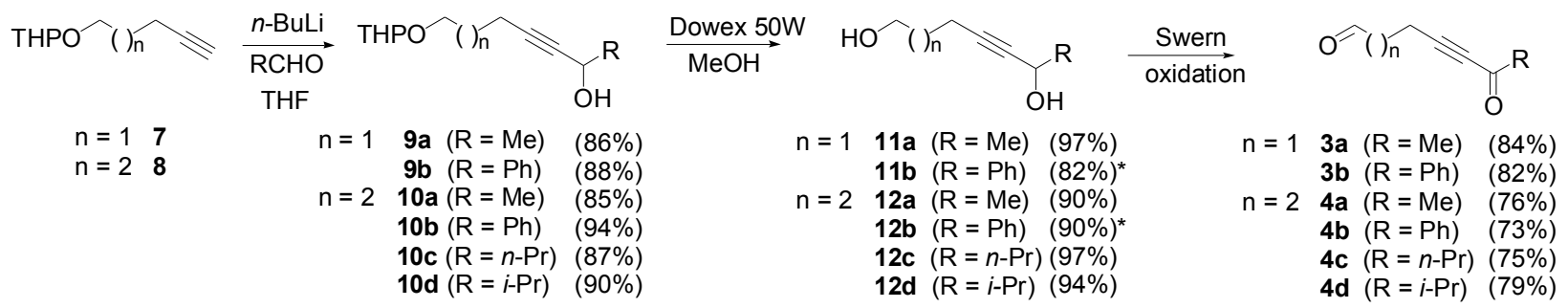

*: calculated yields based on reacted starting material.

\section{Scheme 2}


With the required linear precursors in hands, we turned our attention to their condensation with $(S)$-phenylglycinol. The results obtained starting from the various substituted ketoaldehydes are summarized in Table 1.

Table 1. Synthesis of oxazolidine $\beta$-enamino ketones 5-6 by condensation of keto aldehydes 3-4 with $(S)$-phenylglycinol

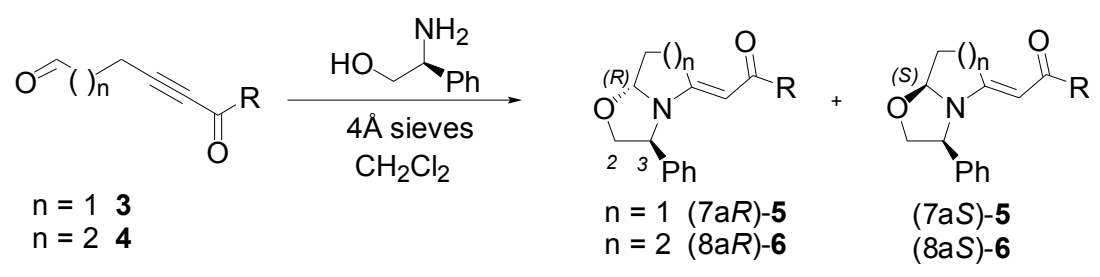

\begin{tabular}{cccccc}
\hline Entry & $\mathrm{n}$ & $\mathrm{R}$ & $\mathbf{3}$ or $\mathbf{4}$ & $\begin{array}{c}\text { Ratio }^{\mathrm{a}}(7 \mathrm{a} R)-\mathbf{5}:(7 \mathrm{a} S)-5 \\
\text { or }(8 \mathrm{a} R)-\mathbf{6}:(8 \mathrm{a} S)-\mathbf{6}\end{array}$ & 5 or 6 $(Y i e l d)^{\mathrm{b}}$ \\
\hline 1 & 1 & $\mathrm{CH}_{3}$ & $\mathbf{3 a}$ & $75: 25$ & $(7 \mathrm{a} R)-\mathbf{5 a}(48 \%)$ \\
& & & & & $(7 \mathrm{a} S)-5 \mathbf{a}(18 \%)$ \\
2 & 1 & $\mathrm{Ph}$ & $\mathbf{3 b}$ & $85: 15$ & $(7 \mathrm{a} R)-\mathbf{5 b}(66 \%)$ \\
& & & & & $(7 \mathrm{a} S)-\mathbf{5 b}(11 \%)$ \\
3 & 2 & $\mathrm{CH}_{3}$ & $\mathbf{4 a}$ & $100: 0$ & $(8 \mathrm{a} R)-\mathbf{6 a}(62 \%)$ \\
4 & 2 & $\mathrm{Ph}_{5}$ & $\mathbf{4 b}$ & $100: 0$ & $(8 \mathrm{a} R)-\mathbf{6 b}(72 \%)$ \\
6 & 2 & $-\left(\mathrm{CH}_{2}\right)_{2} \mathrm{CH}_{3}$ & $\mathbf{4 c}$ & $100: 0$ & $(8 \mathrm{a} R)-\mathbf{6 c}(70 \%)$ \\
\hline
\end{tabular}

adetermined by GC and NMR. ${ }^{\mathrm{b}}$ Isolated yields.

When reacted in $\mathrm{CH}_{2} \mathrm{Cl}_{2}$ in the presence of the chiral amine and $4 \AA$ molecular sieves at room temperature, ketoaldehyde 3a afforded the expected chiral $\beta$-enamino ketone 5a as a mixture of epimers at $\mathrm{C}-7 \mathrm{a}$ in a 75:25 ratio (estimated by GC and NMR) and 66\% overall yield (Table 1, entry 1). Column chromatography readily afforded the isolation of the two diastereomers in respectively $48 \%$ and $18 \%$ yields. The configuration at $\mathrm{C}-7 \mathrm{a}$ of the major isomer of $\mathbf{5 a}$ was assigned to $(R)$, by analogy with analogous pyrrolidine $\beta$-enaminoester $\mathbf{1}^{8}$ (Scheme 1 ), based on the comparison of their chemical shifts in ${ }^{13} \mathrm{C}$ NMR. In particular, similar chemical shifts for C-2 and C-3 were observed for the major isomer of $\mathbf{5 a}$ and for $(7 \mathrm{a} R)-\mathbf{1}$. In contrast, the $(7 \mathrm{a} S)$ minor isomer of 5a displayed very different chemical shifts (Table 2).

Likewise, when reacted with $(S)$-phenylglycinol the phenylketone $\mathbf{3 b}$ gave rise to a $85: 15$ mixture of $\beta$-enaminoketones (7aR)-5b and (7aS)-5b that were subsequently isolated in $66 \%$ and $11 \%$ respective yields (Table 1 , entry 2 ). The absolute configurations of both isomers were assigned as above by comparison of the chemical shifts of C-2 and C-3 (Table 2). It was of note that the minor diastereomer (7aS)-5b slowly isomerized in $\mathrm{CDCl}_{3}$ solution into (7aR)-5b, which in turn evolved to the corresponding pyrrole derivative as substantiated by the characteristic ${ }^{1} \mathrm{H}$ 
NMR aromatic signals at $6.05,6.24$ and $6.95 \mathrm{ppm}$. However, the ratio of the two diastereomers does not evolve after prolonged reaction time in $\mathrm{CH}_{2} \mathrm{Cl}_{2}$.

Table 2. Characteristic ${ }^{13} \mathrm{C}$ NMR chemical shifts for oxazolo piperidines $\mathbf{1}$ and $\mathbf{5}$

\begin{tabular}{ccc}
\hline Compounds & $\delta$ C-2 $(\mathrm{ppm})$ & $\delta \mathrm{C}-3(\mathrm{ppm})$ \\
\hline$(7 \mathrm{a} R)-\mathbf{1}$ & 76.3 & 63.4 \\
$(7 \mathrm{a} R)-\mathbf{5 a}$ & 76.3 & 62.9 \\
$(7 \mathrm{a} R)-\mathbf{5 b}$ & 76.0 & 62.4 \\
$(7 \mathrm{a} S)-5 \mathbf{a}$ & 78.8 & 58.9 \\
$(7 \mathrm{a} S)-\mathbf{5 b}$ & 79.2 & 59.2 \\
\hline
\end{tabular}

As for the homologous ketoaldehydes 4a-d, their condensation with (S)-phenylglycinol afforded the corresponding piperidine $\beta$-enaminoketones 6a-d respectively as single isomers, in high isolated yields (Table 1, entries 3-6). X-ray analysis ${ }^{14}$ performed on crystalline $\mathbf{6 b}$ allowed us to assign the $(8 \mathrm{a} R)$ absolute configuration. The same stereochemistry was attributed to piperidine compounds 6a, $\mathbf{6 c}$ and $\mathbf{6 d}$, based on the comparison of ${ }^{13} \mathrm{C}$ NMR spectroscopic data. Indeed, compounds 6a-d and the piperidine $\beta$-enaminoester (8a $R$ )-2 displayed similar chemical shifts for C-2 and C-3 (Table 3).

Table 3. Characteristic ${ }^{13} \mathrm{C}$ NMR chemical shifts for oxazolo piperidines $\mathbf{2}$ and $\mathbf{6}$

\begin{tabular}{ccc}
\hline Compounds & $\delta$ C-2 $(\mathrm{ppm})$ & $\delta \mathrm{C}-3(\mathrm{ppm})$ \\
\hline$(8 \mathrm{a} R)-\mathbf{2}$ & 73.4 & 61.6 \\
$(8 \mathrm{a} R)-\mathbf{6 a}$ & 73.4 & 61.5 \\
$(8 \mathrm{a} R)-\mathbf{6 b}$ & 73.7 & 62.3 \\
$(8 \mathrm{a} R)-\mathbf{6 c}$ & 73.4 & 61.6 \\
$(8 \mathrm{a} R)-\mathbf{6 d}$ & 73.5 & 61.9 \\
\hline
\end{tabular}

In the above study, we demonstrated that the present methodology consisting in the condensation of a chiral amine with alkynoates could be successfully extended to alkynones, allowing the efficient synthesis of the target pyrrolidine and piperidine $\beta$-enamino ketones. Noteworthy, as conjugated alkynones reacted much faster than the corresponding alkynoates, the follow-up of the reaction by NMR experiment that would give clues on the involved intermediates proved impossible. As for the ester analogue, piperidine derivatives 6a-d were obtained with excellent diastereoselectivities. In contrast, poorer diastereomeric excesses were obtained for pyrrolidine compounds $\mathbf{5 a}$ and $\mathbf{5 b}$ (d.e. 50 to $70 \%$ ) for unclear reasons.

Reduction of $\beta$-enamino ketones may lead to $\gamma$-amino alcohols some of which are interesting for their biological and pharmaceutical properties as well as their wide application in synthesis. ${ }^{15}$ In this context, the previously synthesized chiral $\beta$-enamino ketones appear as convenient precursors of chiral pyrrolidine and piperidine $\gamma$-amino alcohols. In this work, we turned our 
attention towards the total synthesis of 2-(2-hydroxypropyl)-1-methylpyrrolidine (13) (Scheme 3 ) whose four enantiomers have been described, ${ }^{16}$ three of them being natural products. Starting from enantiopure pyrrolidine $\beta$-enaminoketone ( $7 \mathrm{a} R$ )-5a, the key step of our synthesis relied on a diastereoselective reduction. The absolute configurations of the two newly created stereogenic centers were to be assigned based on the identification of the final generated product(s). Various methods including catalytic hydrogenations, ${ }^{4 \mathrm{~b}}$ dissolving metal reduction, ${ }^{17}$ and treatment with $\mathrm{LiBH}_{4} / \mathrm{CeCl}_{3}{ }^{18}$ or with $\mathrm{NaBH}_{4}$ in glacial acetic acid ${ }^{19}$ have been described to afford predominantly syn amino alcohols. We decided to perform the reduction with in situ generated sodium triacetoxy borohohydride in acetic acid, as this method had been successfully used in our laboratory to reduce pyrrolidine $\beta$-enamino ester 1 into the corresponding $\beta$-amino ester with a high diastereoselectivity. ${ }^{3 \mathrm{c}}$ To our delight, these reaction conditions applied to compound $(7 \mathrm{a} R)$ 5a cleanly led to the reduction of the $\mathrm{C}-\mathrm{C}$ double bond and of the ketone moiety along with the cleavage of the oxazolidine ring to give pyrrolidine diol 14, as a single isomer according to NMR. Noteworthy, the reduction performed on isomer $(7 \mathrm{aS})-\mathbf{5 a}$ yielded to the same diastereomer 14, showing that the lack of diastereoselectivity during the formation of the oxazolidine 5a is of little importance in this case. Compound $\mathbf{1 4}$ was submitted to debenzylation $\left(\mathrm{H}_{2}, \mathrm{Pd}(\mathrm{OH})_{2} / \mathrm{C}\right)$ followed by the in situ carbamatation in the presence of $\mathrm{Boc}_{2} \mathrm{O}$, to give amino alcohol 15. Unfortunately, column chromatography did not allow the separation of the latter from 2-phenylethanol. To allow easier isolation, compound $\mathbf{1 4}$ was thus subjected to a bisacetylation to give pyrrolidine acetate $\mathbf{1 6}$ in $46 \%$ overall yield from 5a. Debenzylation of compound $16\left(\mathrm{H}_{2}, \mathrm{Pd}(\mathrm{OH})_{2} / \mathrm{C}\right)$ followed by the in situ carbamatation in the presence of $\mathrm{Boc}_{2} \mathrm{O}$, gave rise to acetate 17 in $85 \%$ yield. Treatment with lithium aluminium hydride led to the simultaneous reduction of the carbamate and to the deprotection of the alcohol function to yield the expected compound $\mathbf{1 3}$ in $88 \%$ yield. The spectroscopic data ${ }^{16,20}$ and the optical rotation of this compound $\left\{[\alpha]_{\mathrm{D}}{ }^{24}-50(\mathrm{c} 1.28, \mathrm{MeOH})\right\}$ were identical with those reported in the literature for the $\left(2 R, 2^{\prime} R\right)-13$ diastereomer $\left\{[\alpha]_{\mathrm{D}}{ }^{22}-49(\mathrm{c} 0.4, \mathrm{EtOH})^{20} ;[\alpha]_{\mathrm{D}}{ }^{20}-50.2(\mathrm{c} 0.466, \mathrm{EtOH})^{21}\right.$; $\left.[\alpha]_{\mathrm{D}}{ }^{25}-53(\mathrm{c} 1.025, \mathrm{EtOH})^{16}\right\},(-)$-hygroline, an alkaloid isolated from Eryhroxylum coca. ${ }^{22}$ This result allowed us to assign the $\left(2 R, 2^{\prime} R\right)$ absolute stereochemistry to compounds 14-17 (Scheme 3).

As for mechanistic considerations, the observation that both $(7 \mathrm{a} R)$ and $(7 \mathrm{a} S)$ isomers of compound 5a were reduced into the same diastereomer showed that the geometry of the ring fusion did not control the stereochemistry at the $\mathrm{C}-2$ center of pyrrolidine 14. So, we reasoned that the control of the stereochemistry of the latter was induced by the chiral center bearing the phenyl substituent, the oxazolidine moiety being initially cleaved or not. The key step of the reaction would then consist in the reduction of the iminium moiety of intermediate boro enolates $\mathbf{I}$ or $\mathbf{I I}^{23}$ via an hydride transfer from the less hindered face (Re face at $\mathrm{C}-5$ or $\mathrm{C}-2$ respectively) anti to the phenyl substituent (Scheme 4). In our scenario, subsequent reduction of the resulting ketone moiety of intermediates III or IV would lead to a syn 1,3-amino alcohol, as previously reported for the reduction of linear $\beta$-enaminoketones. ${ }^{20}$ 


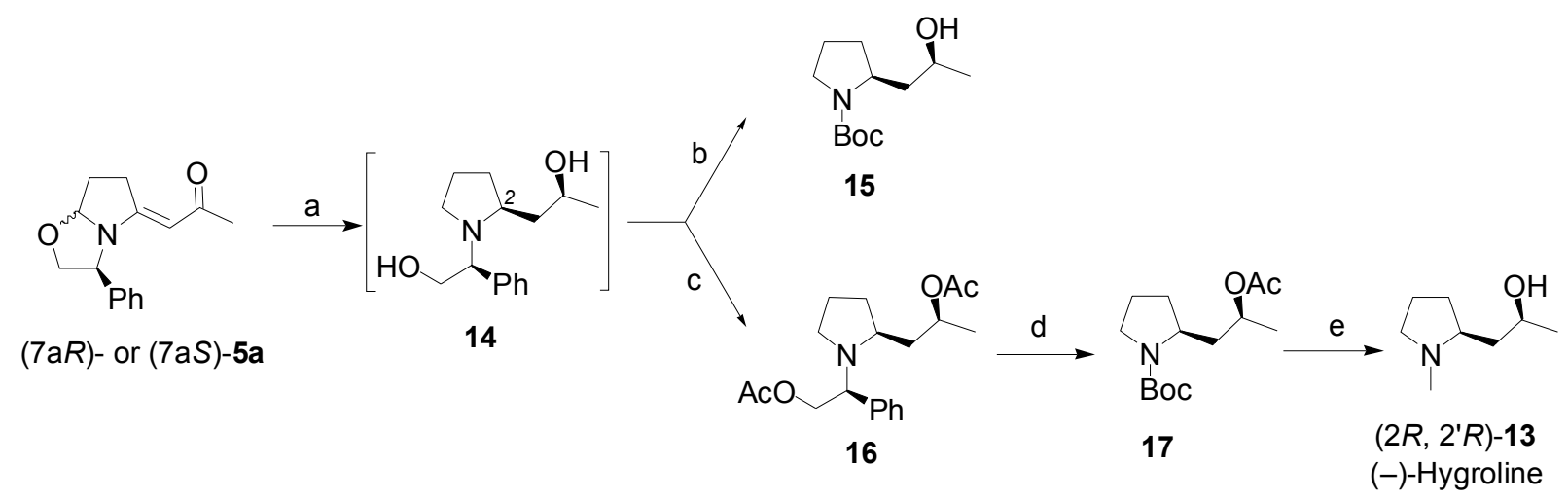

Scheme 3. Reagent and conditions: (a) $\mathrm{NaBH}_{4}, \mathrm{AcOH}, \mathrm{CH}_{3} \mathrm{CN}$; (b) $\mathrm{H}_{2}, \mathrm{Pd}(\mathrm{OH})_{2} / \mathrm{C}, \mathrm{Boc}_{2} \mathrm{O}$, $\mathrm{AcOMe}, 63 \%$ (calculated yield for 2 steps); (c) $\mathrm{Ac}_{2} \mathrm{O}, \mathrm{NEt}_{3}, \mathrm{DMAP}, \mathrm{CH}_{2} \mathrm{Cl}_{2}, 46 \%$ (2 steps); (d) $\mathrm{H}_{2}, \mathrm{Pd}(\mathrm{OH})_{2} / \mathrm{C}, \mathrm{Boc}_{2} \mathrm{O}$, AcOMe, $85 \%$; (e) $\mathrm{LiAlH}_{4}, \mathrm{THF}, 88 \%$.

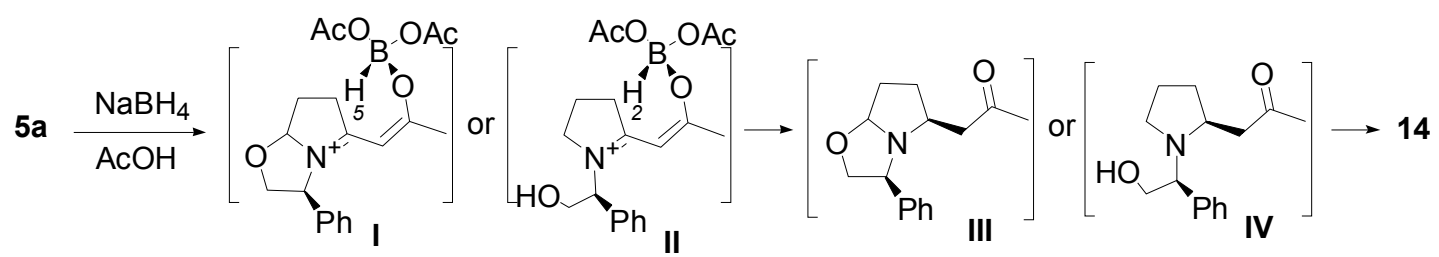

\section{Scheme 4}

\section{Conclusions}

In conclusion, we developed a valuable methodology for the synthesis of chiral oxazolo pyrrolidine and piperidine $\beta$-enamino ketones by condensation of $(S)$-phenylglycinol with $\omega$-oxo alkynones. The synthetic potential of these compounds was illustrated by the total enantioselective synthesis of alkaloid (-)-hygroline.

\section{Experimental Section}

General Procedures. Unless otherwise specified, materials were purchased from commercial suppliers and used without further purification. THF was distilled from sodium/ benzophenone ketyl immediately prior to use. $\mathrm{CH}_{2} \mathrm{Cl}_{2}$ was distilled from calcium hydride. All reactions were carried out under argon. Thin layer chromatography analyses were performed on Merck precoated silica gel $\left(60 \mathrm{~F}_{254}\right)$ plates and column chromatography on silica gel Gerudan SI 60 (40$60 \mu \mathrm{m})$ (Merck). Melting points are uncorrected. IR: Philips PU 9700. Gas chromatographies were performed on a capillary Chrompack CP-SIL5. Optical rotation: Perkin-Elmer 241 polarimeter. Elemental analysis: Service de Microanalyse de l'ICSN (Gif sur Yvette). HMRS 
were recorded on a JEOL MS 700 mass spectrometer and a Thermo Electron Orbitrap mass spectrometer. NMR: Bruker ARX 250 spectrometer $\left(250 \mathrm{MHz}\right.$ and $62.9 \mathrm{MHz}$ for ${ }^{1} \mathrm{H}$ and ${ }^{13} \mathrm{C}$, respectively). Spectra were recorded in $\mathrm{CDCl}_{3}$ as solvent. Chemical shifts $(\delta)$ were expressed in ppm relative to TMS at $\delta=0$ for ${ }^{1} \mathrm{H}$ and to $\mathrm{CDCl}_{3}$ at $\delta=77.16$ for ${ }^{13} \mathrm{C}$ and coupling constants $(J)$ in Hertz.

\section{General procedure for the preparation of compounds 9a-b and 10a-d}

To a solution of alkyne 7 or $8(10 \mathrm{mmol})$ in anhydrous THF $(40 \mathrm{~mL})$ at $-78{ }^{\circ} \mathrm{C}$ was added dropwise $n$-BuLi $(2.5 \mathrm{M}$ in hexanes, 1.1 equiv). The reaction mixture was stirred at this temperature for $30 \mathrm{~min}$ and the required aldehyde (3 equiv of acetaldehyde and 1.1 equiv of the other aldehydes) was subsequently added dropwise. The reaction mixture was allowed to warm to room temperature and after stirring for $5 \mathrm{~h}$, quenched with a saturated aqueous $\mathrm{NH}_{4} \mathrm{Cl}$ solution $(20 \mathrm{~mL})$. The solvent was removed in vacuo and the aqueous layer was extracted with $\mathrm{CH}_{2} \mathrm{Cl}_{2}$ $(5 \times 20 \mathrm{~mL})$. The combined organic layers were washed with water and brine, dried over $\mathrm{Na}_{2} \mathrm{SO}_{4}$ and concentrated in vacuo. Silica gel column chromatography of the residue (AcOEt:cyclohexane 2:8) afforded the pure expected compounds as oils.

7-(Tetrahydro-2H-pyran-2-yloxy)hept-3-yn-2-ol (9a). Colorless oil (86\%); IR (neat) 3400, $2250 \mathrm{~cm}^{-1} ;{ }^{1} \mathrm{H}$ NMR $\left(\mathrm{CDCl}_{3}\right) \delta 1.42(\mathrm{~d}, J=6.5 \mathrm{~Hz}, 3 \mathrm{H}), 1.50-1.85(\mathrm{~m}, 8 \mathrm{H}), 2.03(\mathrm{br} \mathrm{s}, 1 \mathrm{H}), 2.33$ (dt, $J=2$ and $7 \mathrm{~Hz}, 2 \mathrm{H}), 3.43-3.54(\mathrm{~m}, 2 \mathrm{H}), 3.77-3.87(\mathrm{~m}, 2 \mathrm{H}), 4.48-4.53(\mathrm{~m}, 1 \mathrm{H}), 4.59-4.61$ (m, 1H); ${ }^{13} \mathrm{C} \mathrm{NMR}\left(\mathrm{CDCl}_{3}\right) \delta 15.5,19.3,24.6,25.4,28.7,30.5,58.1,62.0,65.8,82.8,83.3,98.6$; HRMS (ESI ${ }^{+}$) calcd for $\mathrm{C}_{12} \mathrm{H}_{20} \mathrm{O}_{3} \mathrm{Na}(\mathrm{M}+\mathrm{Na})^{+}: 235.1305$, found: 235.1304 .

1-Phenyl-6-(tetrahydro-2H-pyran-2-yloxy)hex-2-yn-1-ol (9b). Colorless oil (88\%); IR (neat) 3400, 2240, $2200 \mathrm{~cm}^{-1}$; ${ }^{1} \mathrm{H}$ NMR $\left(\mathrm{CDCl}_{3}\right) \delta 1.47-1.88(\mathrm{~m}, 8 \mathrm{H}), 2.35-2.43(\mathrm{~m}, 3 \mathrm{H}), 3.43-3.52$ (m, 2H), 3.79-3.88 (m, 2H), $4.58(\mathrm{t}, J=3 \mathrm{~Hz}, 1 \mathrm{H}), 5.43-5.45(\mathrm{~m}, 1 \mathrm{H}), 7.31-7.56(\mathrm{~m}, 5 \mathrm{H}) ;{ }^{13} \mathrm{C}$ NMR $\left(\mathrm{CDCl}_{3}\right) \delta 15.6,19.2,25.3,28.5,30.4,61.9,64.3,65.7,80.6,86.2,98.5,126.5,127.9$, 128.3, 141.4; HRMS ( $\left.\mathrm{ESI}^{+}\right)$calcd for $\mathrm{C}_{17} \mathrm{H}_{22} \mathrm{O}_{3} \mathrm{Na}(\mathrm{M}+\mathrm{Na})^{+}: 297.1461$, found: 297.1460.

8-(Tetrahydro-2H-pyran-2-yloxy)oct-3-yn-2-ol (10a). Colorless oil (85\%); IR (neat) 3400, $2220 \mathrm{~cm}^{-1} ;{ }^{1} \mathrm{H}$ NMR $\left(\mathrm{CDCl}_{3}\right) \delta 1.42(\mathrm{~d}, J=6.5 \mathrm{~Hz}, 3 \mathrm{H}), 1.49-1.82(\mathrm{~m}, 10 \mathrm{H}), 2.03(\mathrm{br} \mathrm{s}, 1 \mathrm{H})$, $2.24(\mathrm{dt}, J=2$ and $7 \mathrm{~Hz}, 2 \mathrm{H}), 3.37-3.53(\mathrm{~m}, 2 \mathrm{H}), 3.72-3.87(\mathrm{~m}, 2 \mathrm{H}), 4.48-4.52(\mathrm{~m}, 1 \mathrm{H}), 4.57-$ $4.60(\mathrm{~m}, 1 \mathrm{H}) ;{ }^{13} \mathrm{C}$ NMR $\left(\mathrm{CDCl}_{3}\right) \delta 18.6,19.7,24.8,25.5,25.6,29.0,30.8,58.6,62.4,67.0,82.7$, 84.4; 98.9; HRMS (ESI ${ }^{+}$) calcd for $\mathrm{C}_{13} \mathrm{H}_{22} \mathrm{O}_{3} \mathrm{Na}(\mathrm{M}+\mathrm{Na})^{+}: 249.1461$, found: 249.1460 .

1-Phenyl-7-(tetrahydro-2H-pyran-2-yloxy)hep-2-yn-1-ol (10b). Colorless oil (94\%); IR (neat) $3400,2230 \mathrm{~cm}^{-1} ;{ }^{1} \mathrm{H}$ NMR $\left(\mathrm{CDCl}_{3}\right) \delta 1.49-1.82(\mathrm{~m}, 10 \mathrm{H}), 2.28-2.33(\mathrm{~m}, 2 \mathrm{H}), 2.65$ (br s, $1 \mathrm{H}), 3.35-3.50(\mathrm{~m}, 2 \mathrm{H}), 3.70-3.86(\mathrm{~m}, 2 \mathrm{H}), 4.56(\mathrm{t}, J=3 \mathrm{~Hz}, 1 \mathrm{H}), 5.42(\mathrm{~s}, 1 \mathrm{H}), 7.26-7.54(\mathrm{~m}$, $5 \mathrm{H}) ;{ }^{13} \mathrm{C} \mathrm{NMR}\left(\mathrm{CDCl}_{3}\right) \delta 18.8,19.6,25.4,25.5,29.0,30.7,62.3,64.7,67.0,80.5,87.1,98.8$, 126.7, 128.2, 128.6, 141.4; HRMS (ESI $\left.{ }^{+}\right)$calcd for $\mathrm{C}_{18} \mathrm{H}_{24} \mathrm{O}_{3} \mathrm{Na}(\mathrm{M}+\mathrm{Na})^{+}: 311.1617$, found: 311.1618 .

10-(Tetrahydro-2H-pyran-2-yloxy)dec-5-yn-4-ol (10c). Colorless oil (87\%); IR (neat) 3400 $\mathrm{cm}^{-1} ;{ }^{1} \mathrm{H}$ NMR $\left(\mathrm{CDCl}_{3}\right) \delta 0.92(\mathrm{t}, J=7.25 \mathrm{~Hz}, 3 \mathrm{H}), 1.39-1.73(\mathrm{~m}, 14 \mathrm{H}), 2.03(\mathrm{~s}, 1 \mathrm{H}), 2.23(\mathrm{dt}, J$ $=6.5$ and $1.5 \mathrm{~Hz}, 2 \mathrm{H}), 3.37-3.50(\mathrm{~m}, 2 \mathrm{H}), 3.69-3.84(\mathrm{~m}, 2 \mathrm{H}) ; 4.30-4.57(\mathrm{~m}, 1 \mathrm{H}), 4.55-4.58(\mathrm{~m}$, 
$1 \mathrm{H}) ;{ }^{13} \mathrm{C} \mathrm{NMR}\left(\mathrm{CDCl}_{3}\right) \delta 13.9,18.6,19.7,25.5,29.0,30.8,40.4,62.4,62.5,67.1,81.8,85.1$, 98.9; HRMS (ESI ${ }^{+}$) calcd for $\mathrm{C}_{15} \mathrm{H}_{26} \mathrm{O}_{3} \mathrm{Na}(\mathrm{M}+\mathrm{Na})^{+}: 277.1774$, found: 277.1772 .

2-Methyl-9-(tetrahydro-2H-pyran-2-yloxy)non-4-yn-3-ol (10d). Colorless oil (90\%); IR (neat) $3400 \mathrm{~cm}^{-1} ;{ }^{1} \mathrm{H}$ NMR $\left(\mathrm{CDCl}_{3}\right) \delta 0.96(\mathrm{~d}, J=6.75 \mathrm{~Hz}, 3 \mathrm{H}), 0.99(\mathrm{~d}, J=6.75 \mathrm{~Hz}, 3 \mathrm{H}), 1.51-$ $1.90(\mathrm{~m}, 12 \mathrm{H}), 2.26(\mathrm{dt}, J=2$ and $7 \mathrm{~Hz}, 2 \mathrm{H}), 3.37-3.53(\mathrm{~m}, 2 \mathrm{H}), 3.72-3.86(\mathrm{~m}, 2 \mathrm{H}), 4.13-4.16$ $(\mathrm{m}, 1 \mathrm{H}), 4.57-4.60(\mathrm{~m}, 1 \mathrm{H}) ;{ }^{13} \mathrm{C} \mathrm{NMR}\left(\mathrm{CDCl}_{3}\right) \delta 17.5,18.2,18.6,19.6,25.5,28.9,30.7,34.7$, $62.2,67.0,68.0,80.3,85.6,98.8$; HRMS $\left(\mathrm{ESI}^{+}\right)$calcd for $\mathrm{C}_{15} \mathrm{H}_{26} \mathrm{O}_{3} \mathrm{Na}(\mathrm{M}+\mathrm{Na})^{+}:$277.1774, found: 277.1774 .

\section{General procedure for the deprotection of compounds 9a-b and 10a-d}

A solution of the substrate $(10 \mathrm{mmol})$ in $\mathrm{MeOH}(60 \mathrm{~mL})$ was stirred at room temperature in the presence of Dowex 50W (2.5 g). The resulting reaction was monitored by tlc or GC (reaction time: $6 \mathrm{~h}$ for $9 \mathbf{a}$ and 10a,c,d and $4 \mathrm{~h}$ for $\mathbf{9 b}$ and 10b). The reaction mixture was filtered and concentrated in vacuo. Silica gel column chromatography (AcOEt:cyclohexane 1:1) afforded pure diols as oils.

Hept-4-yne-1,6-diol (11a). Colorless oil (97\%); IR (neat) 3340, $2260 \mathrm{~cm}^{-1} ;{ }^{1} \mathrm{H}$ NMR $\left(\mathrm{CDCl}_{3}\right)$ $\delta 1.43(\mathrm{dd}, J=1$ and $6.5 \mathrm{~Hz}, 3 \mathrm{H}), 1.71-1.81(\mathrm{~m}, 2 \mathrm{H}), 2.31-2.40(\mathrm{~m}, 4 \mathrm{H}), 3.76(\mathrm{t}, J=6 \mathrm{~Hz}, 2 \mathrm{H})$, 4.47-4.53 (m, 1H); ${ }^{13} \mathrm{C} \mathrm{NMR}\left(\mathrm{CDCl}_{3}\right) \delta 15.1,24.5,31.0,58.0,60.9,82.9,83.4$; HRMS $\left(\mathrm{ESI}^{+}\right)$ calcd for $\mathrm{C}_{7} \mathrm{H}_{12} \mathrm{O}_{2} \mathrm{Na}(\mathrm{M}+\mathrm{Na})^{+}: 151.0729$, found: 151.0727 .

1-Phenylhex-2-yne-1,6-1-diol (11b). Colorless oil (74\% along with $10 \%$ recovered starting material 9b). The spectroscopic data are in accordance with that reported in the literature. ${ }^{10}$

Oct-5-yne-1,7-diol (12a). Colorless oil (90\%); IR (neat) 3320, 2250, $2220 \mathrm{~cm}^{-1}$; ${ }^{1} \mathrm{H}$ NMR $\left(\mathrm{CDCl}_{3}\right) \delta 1.41(\mathrm{~d}, J=6.5 \mathrm{~Hz}, 3 \mathrm{H}), 1.54-1.70(\mathrm{~m}, 4 \mathrm{H}), 2.21-2.66(\mathrm{~m}, 2 \mathrm{H}), 3.63(\mathrm{t}, J=6 \mathrm{~Hz}$, $2 \mathrm{H}), 3.80(\mathrm{br} \mathrm{s}, 2 \mathrm{H}), 4.45-4.53(\mathrm{~m}, 1 \mathrm{H}) ;{ }^{13} \mathrm{C} \mathrm{NMR}\left(\mathrm{CDCl}_{3}\right) \delta 18.4,24.6,24.8,31.4,58.0,61.8$, 82.8, 83.8; HRMS ( $\left.\mathrm{ESI}^{+}\right)$calcd for $\mathrm{C}_{8} \mathrm{H}_{14} \mathrm{O}_{2} \mathrm{Na}(\mathrm{M}+\mathrm{Na})^{+}: 165.0886$, found: 165.0884 .

1-Phenylhept-2-yne-1,7-diol (12b). Colorless oil (65\% along with $27 \%$ recovered starting material 10b); IR (neat) 3340, 2240, $2200 \mathrm{~cm}^{-1} ;{ }^{1} \mathrm{H}$ NMR $\left(\mathrm{CDCl}_{3}\right) \delta 1.55-1.71(\mathrm{~m}, 4 \mathrm{H}), 2.25-$ $2.31(\mathrm{~m}, 2 \mathrm{H}), 2.75(\mathrm{br} \mathrm{s}, 2 \mathrm{H}), 3.60(\mathrm{t}, J=6 \mathrm{~Hz}, 2 \mathrm{H}), 5.41(\mathrm{~s}, 1 \mathrm{H}), 7.26-7.53(\mathrm{~m}, 5 \mathrm{H}) ;{ }^{13} \mathrm{C} \mathrm{NMR}$ $\left(\mathrm{CDCl}_{3}\right) \delta 18.7,24.9,31.7,62.2,64.7,80.6,87.1,126.7,128.2,128.6,141.4$; HRMS (ESI $\left.{ }^{+}\right)$ calcd for $\mathrm{C}_{13} \mathrm{H}_{16} \mathrm{O}_{2} \mathrm{Na}(\mathrm{M}+\mathrm{Na})^{+}$: 227.1042, found: 227.1041 .

Dec-5-yne-1,7-diol (12c). Colorless oil (97\%); IR (neat) $3340 \mathrm{~cm}^{-1} ;{ }^{1} \mathrm{H}$ NMR $\left(\mathrm{CDCl}_{3}\right) \delta 0.91$ (t, $J=7.25 \mathrm{~Hz}, 3 \mathrm{H}), 1.38-1.50(\mathrm{~m}, 2 \mathrm{H}), 1.52-1.68(\mathrm{~m}, 6 \mathrm{H}), 2.22(\mathrm{dt}, J=7 \mathrm{and} 2 \mathrm{~Hz}, 2 \mathrm{H}), 2.59$ (br s, $2 \mathrm{H}), 3.63(\mathrm{t}, J=6.25 \mathrm{~Hz}, 2 \mathrm{H}), 4.31(\mathrm{td}, J=6.5$ and $2 \mathrm{~Hz}, 1 \mathrm{H}) ;{ }^{13} \mathrm{C} \mathrm{NMR}\left(\mathrm{CDCl}_{3}\right) \delta 13.9,18.5$, 18.6, 25.0, 31.7, 40.3, 62.2, 62.4, 82.0, 84.9; $\mathrm{HRMS}\left(\mathrm{ESI}^{+}\right)$calcd for $\mathrm{C}_{10} \mathrm{H}_{18} \mathrm{O}_{2} \mathrm{Na}(\mathrm{M}+\mathrm{Na})^{+}$: 193.1199, found: 193.1198 .

8-Methylnon-5-yne-1,7-diol (12d). Colorless oil (94\%); IR (neat) $3320 \mathrm{~cm}^{-1} ;{ }^{1} \mathrm{H}$ NMR $\left(\mathrm{CDCl}_{3}\right)$ $\delta 0.95(\mathrm{~d}, J=6.75 \mathrm{~Hz}, 3 \mathrm{H}), 0.97(\mathrm{~d}, J=6.75 \mathrm{~Hz}, 3 \mathrm{H}), 1.55-1.70(\mathrm{~m}, 4 \mathrm{H}), 1.78-1.88(\mathrm{~m}, 3 \mathrm{H})$, 2.23-2.91 (m, 2H), $3.67(\mathrm{t}, J=6 \mathrm{~Hz}, 2 \mathrm{H}), 4.13-4.16(\mathrm{~m}, 1 \mathrm{H}) ;{ }^{13} \mathrm{C} \mathrm{NMR}\left(\mathrm{CDCl}_{3}\right) \delta 17.5,18.2$, 18.4, 24.9, 31.5, 34.6, 61.9, 67.8, 80.3, 85.4; HRMS $\left(\mathrm{ESI}^{+}\right)$calcd for $\mathrm{C}_{10} \mathrm{H}_{18} \mathrm{O}_{2} \mathrm{Na}(\mathrm{M}+\mathrm{Na})^{+}$: 193.1199, found: 193.1200 . 


\section{General procedure for the oxidation of compounds 11a-b and 12a-d}

To a cooled $-78{ }^{\circ} \mathrm{C}$ solution of $(\mathrm{COCl})_{2}\left(16 \mathrm{mmol}, 3.2\right.$ equiv) in dry $\mathrm{CH}_{2} \mathrm{Cl}_{2}(70 \mathrm{~mL})$ was slowly added DMSO (26.5 mmol, 5.3 equiv). The mixture was stirred at this temperature for $15 \mathrm{~min}$, then the required diol (5 mmol, 1 equiv) dissolved in $\mathrm{CH}_{2} \mathrm{Cl}_{2}(10 \mathrm{~mL})$ was added dropwise. The reaction mixture was stirred at $-78^{\circ} \mathrm{C}$ for $30 \mathrm{~min}$, then at $-50{ }^{\circ} \mathrm{C}$ for $30 \mathrm{~min}$ more. The solution was cooled to $-78{ }^{\circ} \mathrm{C}$, and $\mathrm{NEt}_{3}$ (55 mmol, 11 equiv) was added. The reaction mixture was allowed to warm to room temperature over $3 \mathrm{~h}$. Water $(40 \mathrm{~mL})$ was added and the aqueous layer was extracted with $\mathrm{CH}_{2} \mathrm{Cl}_{2}(3 \times 30 \mathrm{~mL})$. The combined organic layer was washed with water and brine, dried over $\mathrm{Na}_{2} \mathrm{SO}_{4}$ and concentrated in vacuo. Silica gel column chromatography of the residue (AcOEt/Cyclohexane 3:7) afforded the pure expected ketoaldehydes as oils.

6-Oxohept-4-ynal (3a). Colorless oil (84\%); IR (neat) 1670, 1720, $2205 \mathrm{~cm}^{-1}$; ${ }^{1} \mathrm{H} \mathrm{NMR}\left(\mathrm{CDCl}_{3}\right)$ $\delta 2.32(\mathrm{~s}, 3 \mathrm{H}), 2.67-2.71(\mathrm{~m}, 2 \mathrm{H}), 2.77-2.79(\mathrm{~m}, 2 \mathrm{H}), 9.80(\mathrm{t}, J=0.5 \mathrm{~Hz}, 1 \mathrm{H})$; ${ }^{13} \mathrm{C} \mathrm{NMR}$ $\left(\mathrm{CDCl}_{3}\right) \delta 11.5,32.3,41.0,81.0,91.3,184.2$, 199.0; HRMS (CI) calcd for $\mathrm{C}_{7} \mathrm{H}_{9} \mathrm{O}_{2}(\mathrm{M}+\mathrm{H})^{+}$: 125.0597, found: 125.0600 .

6-Oxo-6-phenylhex-4-ynal (3b). Pale-yellow oil (82\%); IR (neat) 1630, 1710, 2200, $2250 \mathrm{~cm}^{-1}$; ${ }^{1} \mathrm{H}$ NMR $\left(\mathrm{CDCl}_{3}\right) \delta 2.78-2.92(\mathrm{~m}, 4 \mathrm{H}), 7.27-7.64(\mathrm{~m}, 3 \mathrm{H}), 8.09-8.13(\mathrm{~m}, 2 \mathrm{H}), 9.86(\mathrm{~d}, J=0.5$ $\mathrm{Hz}, 1 \mathrm{H}) ;{ }^{13} \mathrm{C} \mathrm{NMR}\left(\mathrm{CDCl}_{3}\right) \delta 12.0,41.4,79.6,94.0,128.5,129.4,134.0,136.5,177.8,199.0$; HRMS (CI) calcd for $\mathrm{C}_{12} \mathrm{H}_{11} \mathrm{O}_{2}(\mathrm{M}+\mathrm{H})^{+}: 187.0759$, found: 187.0760 .

.7-Oxooct-5-ynal (4a). Pale-yellow oil (76\%); IR (neat) 2220, 1720, $1680 \mathrm{~cm}^{-1}$; ${ }^{1} \mathrm{H}$ NMR $\left(\mathrm{CDCl}_{3}\right) \delta 1.95(\mathrm{q}, J=7 \mathrm{~Hz}, 2 \mathrm{H}), 2.33(\mathrm{~s}, 3 \mathrm{H}), 2.54(\mathrm{t}, J=7 \mathrm{~Hz}, 2 \mathrm{H}), 2.63(\mathrm{t}, J=7 \mathrm{~Hz}, 2 \mathrm{H}), 9.81$ $(\mathrm{d}, J=0.75 \mathrm{~Hz}, 1 \mathrm{H}) ;{ }^{13} \mathrm{C} \mathrm{NMR}\left(\mathrm{CDCl}_{3}\right) \delta 18.1,20.0,32.7,42.3,81.9,92.2,184.6,201.1$; HRMS (CI) calcd for $\mathrm{C}_{8} \mathrm{H}_{11} \mathrm{O}_{2}(\mathrm{M}+\mathrm{H})^{+}:$: 139.0759, found: 139.0757 .

7-Oxo-7-phenylhept-5-ynal (4b). Pale-yellow oil (73\%); IR (neat) 2220, 2180,1725, $1645 \mathrm{~cm}^{-1}$; ${ }^{1} \mathrm{H}$ NMR $\left(\mathrm{CDCl}_{3}\right) \delta 2.00$ (quint, $\left.J=7 \mathrm{~Hz}, 2 \mathrm{H}\right), 2.59(\mathrm{t}, J=7 \mathrm{~Hz}, 2 \mathrm{H}), 2.69(\mathrm{t}, J=7 \mathrm{~Hz}, 2 \mathrm{H})$, 7.45-7.61 (m, 3H), 8.10-8.13 (m, 2H), $9.83(\mathrm{~s}, 1 \mathrm{H}) ;{ }^{13} \mathrm{C} \mathrm{NMR}\left(\mathrm{CDCl}_{3}\right) \delta 18.5,20.3,42.5,80.3$, 94.9, 128.6, 129.6, 134.1, 136.7, 178.0, 201.1; HRMS (CI) calcd for $\mathrm{C}_{13} \mathrm{H}_{13} \mathrm{O}_{2}(\mathrm{M}+\mathrm{H})^{+}$: 201.0916, found: 201.0921 .

7-Oxodec-5-ynal (4c). Yellow oil (75 \%); IR (neat) 1665, 1720, $2200 \mathrm{~cm}^{-1} ;{ }^{1} \mathrm{H} \mathrm{NMR}\left(\mathrm{CDCl}_{3}\right) \delta$ $0.88(\mathrm{t}, J=7.5 \mathrm{~Hz}, 3 \mathrm{H}), 1.63(\mathrm{q}, J=7.5 \mathrm{~Hz}, 2 \mathrm{H}), 1.85(\mathrm{q}, J=7 \mathrm{~Hz}, 2 \mathrm{H}), 2.37-2.48(\mathrm{~m}, 4 \mathrm{H}), 2.58$ $(\mathrm{t}, J=7 \mathrm{~Hz}, 2 \mathrm{H}), 9.75(\mathrm{~s}, 1 \mathrm{H}) ;{ }^{13} \mathrm{C} \mathrm{NMR}\left(\mathrm{CDCl}_{3}\right) \delta 13.4,17.5,18.2,20.1,42.4,47.3,81.4,92.2$, 188.1, 201.1; HRMS (ESI $\left.{ }^{+}\right)$calcd for $\mathrm{C}_{10} \mathrm{H}_{14} \mathrm{O}_{2} \mathrm{Na}(\mathrm{M}+\mathrm{Na})^{+}$: 189.0886, found: 189.0885.

8-Methyl-7-oxonon-5-ynal (4d). Colorless oil (79\%); IR (neat) 1660, 1710, $2180 \mathrm{~cm}^{-1} ;{ }^{1} \mathrm{H}$ $\operatorname{NMR}\left(\mathrm{CDCl}_{3}\right) \delta 1.12(\mathrm{~d}, J=7 \mathrm{~Hz}, 3 \mathrm{H}), 1.13(\mathrm{~d}, J=7 \mathrm{~Hz}, 3 \mathrm{H}), 1.87(\mathrm{q}, J=7 \mathrm{~Hz}, 2 \mathrm{H}), 2.42(\mathrm{t}, J$ $=7 \mathrm{~Hz}, 2 \mathrm{H}), 2.54-2.62(\mathrm{~m}, 3 \mathrm{H}), 9.75(\mathrm{~d}, J=1 \mathrm{~Hz}, 1 \mathrm{H}) ;{ }^{13} \mathrm{C} \mathrm{NMR}\left(\mathrm{CDCl}_{3}\right) \delta 17.9,18.3,20.2$, 42.4, 42.9, 80.4, 93.2, 192.1, 201.1; HRMS (ESI ${ }^{+}$) calcd for $\mathrm{C}_{10} \mathrm{H}_{14} \mathrm{O}_{2} \mathrm{Na}(\mathrm{M}+\mathrm{Na})^{+}:$189.0886, found: 189.0888 .

\section{General procedure for the preparation of $5 a-b$ and $6 a-d$}

A mixture of the required ketoaldehyde (5 mmol) 3a-b or 4a-d in $\mathrm{CH}_{2} \mathrm{Cl}_{2}(50 \mathrm{~mL})$, $(S)$ phenylglycinol (1.1 equiv) and $4 \AA$ molecular sieves (10 g) was stirred at room temperature for 4 
h. The reaction mixture was filtered over a Celite ${ }^{\circledR}$ pad. The cake was washed with $\mathrm{CH}_{2} \mathrm{Cl}_{2}$ and the combined filtrates were evaporated in vacuo. Silica gel column chromatography (AcOEt/cyclohexane 1:1) allowed the isolation of the expected compounds.

(1E)-1-[(3S,7a $R$ and 7aS)-3-Phenyltetrahydropyrrolo[2,1-b]oxazol-5(6H)-ylidene]acetone (5a). For (7aR)-5a: White solid (48\%); mp 90-91 ${ }^{\circ} \mathrm{C}$ (from cyclohexane); $[\alpha]_{\mathrm{D}}{ }^{24}+359(c 1.18$, $\left.\mathrm{CHCl}_{3}\right)$; IR $\left(\mathrm{CHBr}_{3}\right) 1655 \mathrm{~cm}^{-1} ;{ }^{1} \mathrm{H}$ NMR $\left(\mathrm{CDCl}_{3}\right) \delta 1.98-2.11(\mathrm{~m}, 1 \mathrm{H}), 2.04(\mathrm{~s}, 3 \mathrm{H}), 2.35-2.48$ $(\mathrm{m}, 1 \mathrm{H}), 3.06-3.32(\mathrm{~m}, 1 \mathrm{H}), 3.70-3.83(\mathrm{~m}, 2 \mathrm{H}), 4.55-4.72(\mathrm{~m}, 2 \mathrm{H}), 5.16(\mathrm{~s}, 1 \mathrm{H}), 5.35(\mathrm{dd}, J=$ 4.25 and $6 \mathrm{~Hz}, 1 \mathrm{H}), 7.25-7.43(\mathrm{~m}, 5 \mathrm{H}) ;{ }^{13} \mathrm{C} \mathrm{NMR}\left(\mathrm{CDCl}_{3}\right) \delta 27.8,30.8,33.0,62.9,76.3,95.4$, 96.9, 125.7, 127.9, 129.1, 139.1, 165.9, 196.1. For (7aS)-5a: Yellow solid (18\%); mp $106.5{ }^{\circ} \mathrm{C}$ (from cyclohexane:AcOEt ); $[\alpha]_{\mathrm{D}}{ }^{24}-115$ (c 1.025, $\left.\mathrm{CHCl}_{3}\right)$; IR $\left(\mathrm{CHBr}_{3}\right) 1650 \mathrm{~cm}^{-1} ;{ }^{1} \mathrm{H}$ NMR $\left(\mathrm{CDCl}_{3}\right) \delta 1.71(\mathrm{~s}, 3 \mathrm{H}), 1.88-2.04(\mathrm{~m}, 1 \mathrm{H}), 2.26-2.36(\mathrm{~m}, 1 \mathrm{H}), 2.92-3.07(\mathrm{~m}, 1 \mathrm{H}), 3.76-3.87(\mathrm{~m}$, $1 \mathrm{H}), 4.18(\mathrm{~d}, J=8.25 \mathrm{~Hz}, 1 \mathrm{H}), 4.43-4.53(\mathrm{~m}, 3 \mathrm{H}), 5.32(\mathrm{dd}, J=5.5$ and $7.5 \mathrm{~Hz}, 1 \mathrm{H}), 7.16-7.34$ $(\mathrm{m}, 5 \mathrm{H}) ;{ }^{13} \mathrm{C} \mathrm{NMR}\left(\mathrm{CDCl}_{3}\right) \delta 27.9,30.1,34.5,58.3,78.8,95.2,96.3,127.1,127.7,128.2,137.9$, 157.3, 194.6; Anal. Calcd for $\mathrm{C}_{15} \mathrm{H}_{17} \mathrm{NO}_{2}$ : C, 74.05; H, 7.04; N, 5.76. Found: C, 73.65; H, 6.91; N, 5.59 .

(2E)-1-Phenyl-2-[(3S,7a $R$ and 7aS)-3-phenyltetrahydropyrrolo[2,1-b]oxazol-5(6H)ylidene]ethanone (5b). For (7aR)-5b: Pale-yellow oil $(66 \%) ;[\alpha]_{\mathrm{D}}{ }^{20}+408\left(c 1.02, \mathrm{CHCl}_{3}\right)$; IR (neat) $1640 \mathrm{~cm}^{-1} ;{ }^{1} \mathrm{H}$ NMR $\left(\mathrm{CDCl}_{3}\right) \delta 2.00-2.15(\mathrm{~m}, 1 \mathrm{H}), 2.38-2.51(\mathrm{~m}, 1 \mathrm{H}), 3.22-3.37(\mathrm{~m}, 1 \mathrm{H})$, 3.77-3.99 (m, 2H), 4.66-4.74 (m, 2H), $5.39(\mathrm{dd}, J=4.5$ and $6 \mathrm{~Hz}, 1 \mathrm{H}), 5.86(\mathrm{~s}, 1 \mathrm{H}), 7.27-7.39$ $(\mathrm{m}, 8 \mathrm{H}), 7.75-7.79(\mathrm{~m}, 2 \mathrm{H}) ;{ }^{13} \mathrm{C} \mathrm{NMR}\left(\mathrm{CDCl}_{3}\right) \delta 27.5,33.4,62.4,76.0,91.2,96.6,125.4,127.0$, 127.6, 127.8, 128.7, 130.7, 138.6, 140.3, 167.5, 188.4. For (7aS)-5b: White solid (11\%); mp 153 ${ }^{\circ} \mathrm{C}$ (from cyclohexane:AcOEt); $[\alpha]_{\mathrm{D}}{ }^{20}-94\left(c 1.04, \mathrm{CHCl}_{3}\right)$; IR $\left(\mathrm{CHBr}_{3}\right) 1630 \mathrm{~cm}^{-1} ;{ }^{1} \mathrm{H} \mathrm{NMR}$ $\left(\mathrm{CDCl}_{3}\right) \delta 1.66-2.08(\mathrm{~m}, 1 \mathrm{H}), 2.32-2.42(\mathrm{~m}, 1 \mathrm{H}), 3.10-3.25(\mathrm{~m}, 1 \mathrm{H}), 3.76-3.87(\mathrm{~m}, 1 \mathrm{H}), 4.26$ $(\mathrm{d}, J=8.75 \mathrm{~Hz}, 1 \mathrm{H}), 4.45-4.57(\mathrm{~m}, 2 \mathrm{H}), 5.04(\mathrm{~s}, 1 \mathrm{H}), 5.38(\mathrm{dd}, J=5.5$ and $7.5 \mathrm{~Hz}, 1 \mathrm{H}), 7.15-$ $7.38(\mathrm{~m}, 10 \mathrm{H}) ;{ }^{13} \mathrm{C} \mathrm{NMR}\left(\mathrm{CDCl}_{3}\right) \delta 28.6,35.6,59.2,79.2,93.2,97.1,127.3,127.9,128.4,129.0$, 130.7, 138.3, 141.1, 159.7, 199.7; Anal. Calcd for $\mathrm{C}_{20} \mathrm{H}_{19} \mathrm{NO}_{2}: \mathrm{C}$, 78.66; H, 6.27; N, 4.59. Found: C, 78.43; H, 6.31; N, 4.45 .

(1E)-1-[(3S,8aR)-3-Phenylhexahydro-5H-[1,3]oxazolo[3,2-a]pyridine-5-ylidene]acetone (6a). Pale-yellow oil $(62 \%) ;[\alpha]_{\mathrm{D}}{ }^{20}+171\left(c\right.$ 1.055, $\left.\mathrm{CHCl}_{3}\right)$; IR $\left(\mathrm{CHBr}_{3}\right) 1630 \mathrm{~cm}^{-1}$; ${ }^{1} \mathrm{H}$ NMR $\left(\mathrm{CDCl}_{3}\right) \delta 1.48-1.66(\mathrm{~m}, 2 \mathrm{H}), 1.85(\mathrm{~s}, 3 \mathrm{H}), 1.93-2.03(\mathrm{~m}, 1 \mathrm{H}), 2.30-2.46(\mathrm{~m}, 1 \mathrm{H}), 2.95-3.10(\mathrm{~m}$, $1 \mathrm{H}), 3.27-3.35(\mathrm{~m}, 1 \mathrm{H}), 3.61(\mathrm{t}, J=8.5 \mathrm{~Hz}, 1 \mathrm{H}), 4.55(\mathrm{t}, J=8.5 \mathrm{~Hz}, 1 \mathrm{H}), 4.75(\mathrm{t}, J=8 \mathrm{~Hz}, 1 \mathrm{H})$, $4.85(\mathrm{~s}, 1 \mathrm{H}), 4.91(\mathrm{dd}, J=4.5$ and $9 \mathrm{~Hz}, 1 \mathrm{H}), 7.19-7.41(\mathrm{~m}, 5 \mathrm{H}) ;{ }^{13} \mathrm{C} \mathrm{NMR}\left(\mathrm{CDCl}_{3}\right) \delta 16.9$, 27.9, 28.1, 31.4, 61.5, 73.4, 89.25, 95.6, 125.7, 127.9, 129.2, 138.6, 158.5, 194.9; HRMS (ESI $\left.{ }^{+}\right)$ calcd for $\mathrm{C}_{16} \mathrm{H}_{20} \mathrm{NO}_{2}(\mathrm{M}+\mathrm{H})^{+}: 258.1488$, found: 258.1487 .

(2E)-1-Phenyl-2-[(3S,8aR)-3-phenylhexahydro-5 $H$-[1,3]oxazolo[3,2-a]pyridine-5-

ylidene]ethanone (6b). White solid $(72 \%) ; \mathrm{mp} 152{ }^{\circ} \mathrm{C}$ (from cyclohexane:AcOEt); $[\alpha]_{\mathrm{D}}{ }^{20}+248$ (c 1.065, $\left.\mathrm{CHCl}_{3}\right)$; IR $\left(\mathrm{CHBr}_{3}\right) 1615 \mathrm{~cm}^{-1} ;{ }^{1} \mathrm{H} \mathrm{NMR}\left(\mathrm{CDCl}_{3}\right) \delta 1.56-1.73(\mathrm{~m}, 2 \mathrm{H}), 2.03-2.09(\mathrm{~m}$, $1 \mathrm{H}), 2.36-2.42(\mathrm{~m}, 1 \mathrm{H}), 3.13-3.28(\mathrm{~m}, 1 \mathrm{H}), 3.41-3.44(\mathrm{~m}, 1 \mathrm{H}), 3.67(\mathrm{t}, J=8.75 \mathrm{~Hz}, 1 \mathrm{H}), 4.61$ (t, $J=8.75 \mathrm{~Hz}, 1 \mathrm{H}), 4.84(\mathrm{t}, J=8.25 \mathrm{~Hz}, 1 \mathrm{H}), 5.05$ (dd, $J=4$ and $9.25 \mathrm{~Hz}, 1 \mathrm{H}), 5.51$ (br s, 1H), 7.23-7.43 (m, 10H); ${ }^{13} \mathrm{C} \mathrm{NMR}\left(\mathrm{CDCl}_{3}\right) \delta 17.3,28.2,28.4,62.3,62.3,73.7,89.7,93.3,126.2$, 
127.3, 128.1, 128.3, 129.5, 130.5, 138.6, 142.1, 160.2, 188.2; HRMS (ESI ${ }^{+}$) calcd for $\mathrm{C}_{21} \mathrm{H}_{22} \mathrm{NO}_{2}$ $(\mathrm{M}+\mathrm{H})^{+}: 320.1645$, found: 320.1643 .

(1E)-1-[(3S,8a $R)-3-P h e n y l h e x a h y d r o-5 H-[1,3]$ oxazolo $[3,2-a]$ pyridine-5-ylidene]pentan-2one (6c). Yellow oil $(70 \%)$; $[\alpha]_{\mathrm{D}}{ }^{20}+175\left(c 1.11, \mathrm{CHCl}_{3}\right)$; IR (neat) $1635 \mathrm{~cm}^{-1} ;{ }^{1} \mathrm{H} \mathrm{NMR}\left(\mathrm{CDCl}_{3}\right)$ $\delta 0.75(\mathrm{t}, J=7.5 \mathrm{~Hz}, 3 \mathrm{H}) ; 1.32-1.67(\mathrm{~m}, 4 \mathrm{H}) ; 1.93-2.07(\mathrm{~m}, 3 \mathrm{H}) ; 2.30-2.35(\mathrm{~m}, 1 \mathrm{H}) ; 3.00-3.11$ $(\mathrm{m}, 1 \mathrm{H}) ; 3.28-3.37(\mathrm{~m}, 1 \mathrm{H}) ; 3.63(\mathrm{t}, J=8.5 \mathrm{~Hz}, 1 \mathrm{H}) ; 4.55(\mathrm{t}, J=8.5 \mathrm{~Hz}, 1 \mathrm{H}) ; 4.75(\mathrm{t}, J=8 \mathrm{~Hz}$, $1 \mathrm{H}) ; 4.83(\mathrm{~s}, 1 \mathrm{H}), 4.89-4.95(\mathrm{~m}, 1 \mathrm{H}), 7.20-7.41(\mathrm{~m}, 5 \mathrm{H}) ;{ }^{13} \mathrm{C} \mathrm{NMR}\left(\mathrm{CDCl}_{3}\right) \delta 13.9,17.0,19.1$, 27.9, 28.1, 46.2, 61.6, 73.4, 89.3, 95.4, 125.8, 127.9, 129.1, 138.7, 158.2, 197.9; HRMS (ESI ${ }^{+}$) calcd for $\mathrm{C}_{18} \mathrm{H}_{24} \mathrm{NO}_{3}(\mathrm{M}+\mathrm{H})^{+}: 286.1802$, found: 286.1800 .

(1E)-3-Methyl-1-[(3S,8a $R)-3-p h e n y l h e x a h y d r o-5 H-[1,3]$ oxazolo[[3,2-a]pyridine-5ylidene]butan-2-one (6d). White solid (75\%); mp $75{ }^{\circ} \mathrm{C}$ (from cyclohexane); IR (neat) 1620 $\mathrm{cm}^{-1} ;[\alpha]_{\mathrm{D}}{ }^{20}+171\left(c 0.955, \mathrm{CHCl}_{3}\right) ;{ }^{1} \mathrm{H} \mathrm{NMR}\left(\mathrm{CDCl}_{3}\right) \delta 0.82(\mathrm{~d}, J=7 \mathrm{~Hz}, 3 \mathrm{H}), 0.88(\mathrm{~d}, J=7$ $\mathrm{Hz}, 3 \mathrm{H}), 1.50-1.62(\mathrm{~m}, 2 \mathrm{H}), 1.95-1.98(\mathrm{~m}, 1 \mathrm{H}), 2.22$ (s, J=7 Hz, 1H), 2.28-2.36 (m, 1H), 3.00$3.10(\mathrm{~m}, 1 \mathrm{H}), 3.28-3.37(\mathrm{~m}, 1 \mathrm{H}), 3.65(\mathrm{t}, J=8.5 \mathrm{~Hz}, 1 \mathrm{H}), 4.54-4.60(\mathrm{~m}, 1 \mathrm{H}), 4.75(\mathrm{t}, J=8 \mathrm{~Hz}$, $1 \mathrm{H}), 4.83(\mathrm{~s}, 1 \mathrm{H}), 4.91-4.96(\mathrm{~m}, 1 \mathrm{H}), 7.21-7.41(\mathrm{~m}, 5 \mathrm{H}) ;{ }^{13} \mathrm{C} \mathrm{NMR}\left(\mathrm{CDCl}_{3}\right) \delta 17.2,19.5,19.8$, 28.0, 28.2, 41.5, 61.9, 73.5, 89.4, 94.1, 125.9, 128.0, 129.2, 138.8, 158.6, 201.9 ; HRMS (ESI $\left.{ }^{+}\right)$ calcd for $\mathrm{C}_{18} \mathrm{H}_{24} \mathrm{NO}_{2}(\mathrm{M}+\mathrm{H})^{+}: 286.1802$, found: 286.1804 .

tert-Butyl (2S)-2-[(2S)-2-hydroxypropyl]pyrrolidine-1-carboxylate (15). A solution of $\mathrm{NaBH}(\mathrm{OAc})_{3}$ was prepared by portionwise addition of $\mathrm{NaBH}_{4}(0.23 \mathrm{~g}, 6 \mathrm{mmol})$ to a mixture of glacial acetic acid $(3.5 \mathrm{~mL}, 60 \mathrm{mmol})$ and $\mathrm{CH}_{3} \mathrm{CN}(1.5 \mathrm{~mL})$ at $0^{\circ} \mathrm{C}$. After hydrogen evolution had ceased (30 min), a solution of $5 \mathbf{a}(0.30 \mathrm{~g}, 1.22 \mathrm{mmol})$ in $\mathrm{CH}_{3} \mathrm{CN}(9 \mathrm{~mL})$ was added. After stirring for $3 \mathrm{~h}$ at room temperature, water $(50 \mathrm{~mL})$ was added and solid $\mathrm{Na}_{2} \mathrm{CO}_{3}$ was added until $\mathrm{pH}=9$. The aqueous layer was extracted with $\mathrm{CH}_{2} \mathrm{Cl}_{2}(3 \times 50 \mathrm{~mL})$ and the combined organic layers were washed with brine, dried over $\mathrm{Na}_{2} \mathrm{SO}_{4}$ and concentrated in vacuo. ${ }^{13} \mathrm{C} \mathrm{NMR}\left(\mathrm{CDCl}_{3}\right)$ of the crude diol 14: $\delta 23.8,24.6,30.1,40.4,53.6,59.6,63.2,65.0,69.1,127.8,128.3,129.3$, 138.5. The residue was dissolved in AcOMe $(30 \mathrm{~mL})$ and subjected to hydrogenation $(1 \mathrm{~atm})$ in the presence of $\mathrm{Pd}(\mathrm{OH})_{2} / \mathrm{C}(0.15 \mathrm{~g})$ and $\mathrm{Boc}_{2} \mathrm{O}(0.52 \mathrm{~g}, 2.4 \mathrm{mmol})$ at room temperature for $12 \mathrm{~h}$. The reaction mixture was filtered, the residue thoroughly washed with methyl acetate and the combined filtrates were concentrated in vacuo. Silica gel column chromatography (AcOEt/cyclohexane 2:8) yielded compound 15 along with unseparable 2-phenylethanol $(0.28 \mathrm{~g}$ overall, 63\% calculated yield for 15). From a mixture with 2-phenylethanol: ${ }^{1} \mathrm{H} \mathrm{NMR}\left(\mathrm{CDCl}_{3}\right)$ $\delta 1.17(\mathrm{~d}, J=6.5 \mathrm{~Hz}, 3 \mathrm{H}), 1.46(\mathrm{~s}, 9 \mathrm{H}), 1.37-1.60(\mathrm{~m}, 3 \mathrm{H}, 1.82-1.96(\mathrm{~m}, 3 \mathrm{H}), 3.29-3.35(\mathrm{~m}$, 2H), 3.68-3.86 (m, 1H), $3.90($ br s, $1 \mathrm{H}), 4.10-4.25(\mathrm{~m}, 1 \mathrm{H}) ;{ }^{13} \mathrm{C} \mathrm{NMR}\left(\mathrm{CDCl}_{3}\right) \delta 22.6,23.5$, 28.5, 31.2, 45.6, 46.5, 53.9, 63.8, 79.9, 156.6.

(1S)-2-\{(2S)-1-[(1S)-2-(Acetoxy)-1-phenylethyl]pyrrolidin-2-yl\}-1-methylethyl acetate (16). A solution of $\mathrm{NaBH}(\mathrm{OAc})_{3}$ was prepared by portionwise addition of $\mathrm{NaBH}_{4}(0.42 \mathrm{~g}, 11.1 \mathrm{mmol})$ to a mixture of glacial acetic acid $(6.4 \mathrm{~mL}, 111 \mathrm{mmol})$ and $\mathrm{CH}_{3} \mathrm{CN}(2.5 \mathrm{~mL})$ at $0^{\circ} \mathrm{C}$. After hydrogen evolution had ceased (30 min), a solution of $5 \mathbf{a}(0.54 \mathrm{~g}, 2.22 \mathrm{mmol})$ in $\mathrm{CH}_{3} \mathrm{CN}(10$ $\mathrm{mL})$ was added. After stirring for $3 \mathrm{~h}$ at room temperature, water $(50 \mathrm{~mL})$ was added and solid 
$\mathrm{Na}_{2} \mathrm{CO}_{3}$ was added until $\mathrm{pH}=9$. The aqueous layer was extracted with $\mathrm{CH}_{2} \mathrm{Cl}_{2}(3 \times 50 \mathrm{~mL})$ and the combined organic layers were washed with brine, dried over $\mathrm{Na}_{2} \mathrm{SO}_{4}$ and concentrated in vacuo. The residue was dissolved in $\mathrm{CH}_{2} \mathrm{Cl}_{2}(12 \mathrm{~mL}), \mathrm{Ac}_{2} \mathrm{O}(1.26 \mathrm{~mL}, 13.3 \mathrm{mmol}), \mathrm{NEt}_{3}(2.15$ $\mathrm{mL}, 15.5 \mathrm{mmol})$ and DMAP (27 $\mathrm{mg}, 0.22 \mathrm{~mol})$ were then added. The reaction mixture was stirred at room temperature for $12 \mathrm{~h}$. A saturated aqueous $\mathrm{NH}_{4} \mathrm{Cl}$ solution $(20 \mathrm{~mL})$ was added and the resulting aqueous layer was extracted with $\mathrm{CH}_{2} \mathrm{Cl}_{2}(3 \times 30 \mathrm{~mL})$. The combined organic layers were washed with brine, dried over $\mathrm{Na}_{2} \mathrm{SO}_{4}$ and concentrated in vacuo. Silica gel column chromatography (AcOEt/cyclohexane 3:7) yielded compound $16(0.34 \mathrm{~g}, 46 \%)$ as a colorless oil. $[\alpha]_{\mathrm{D}}{ }^{24}-24\left(c 0.975, \mathrm{CHCl}_{3}\right) ; \mathrm{IR}$ (neat) $1750 \mathrm{~cm}^{-1} ;{ }^{1} \mathrm{H}$ NMR $\left(\mathrm{CDCl}_{3}\right) \delta 0.99(\mathrm{~d}, J=6.25 \mathrm{~Hz}, 3 \mathrm{H})$; $1.42-1.85(\mathrm{~m}, 6 \mathrm{H}) ; 1.96(\mathrm{~s}, 6 \mathrm{H}) ; 2.56-2.66(\mathrm{~m}, 1 \mathrm{H}), 2.85-2.92(\mathrm{~m}, 2 \mathrm{H}), 3.85-3.92(\mathrm{~m}, 1 \mathrm{H})$, $4.34-4.41(\mathrm{~m}, 1 \mathrm{H}), 4.49-4.56(\mathrm{~m}, 1 \mathrm{H}), 4.70-4.78(\mathrm{~m}, 1 \mathrm{H}), 7.22-7.36(\mathrm{~m}, 5 \mathrm{H})$; ${ }^{13} \mathrm{C} \mathrm{NMR}$ $\left(\mathrm{CDCl}_{3}\right) \delta 19.7,20.6,21.1,23.2,30.2,41.5,49.7,58.7,63.8,64.9,69.4,127.2,128.1,128.2$, 140.4, 170.1, 170.4; Anal. Calcd for $\mathrm{C}_{19} \mathrm{H}_{27} \mathrm{NO}_{4}$ : C, 68.44; H, 8.16; N, 4.20. Found: $\mathrm{C}, 68.53$; $\mathrm{H}$, $8.17 ; \mathrm{N}, 4.28$.

tert-Butyl (2S)-2-[(2S)-2-(acetoxy)propyl]pyrrolidine-1-carboxylate (17). A solution of compound $16(0.32 \mathrm{~g}, 0.96 \mathrm{mmol})$ in methyl acetate $(30 \mathrm{~mL})$ was subjected to hydrogenation (1 atm $)$ in the presence of $\mathrm{Pd}(\mathrm{OH})_{2} / \mathrm{C}(0.016 \mathrm{~g}, 0.5$ equiv in weight $)$ and $\mathrm{Boc}_{2} \mathrm{O}(0.44 \mathrm{~g}, 2 \mathrm{mmol})$, at room temperature for $12 \mathrm{~h}$. The reaction mixture was filtered, the residue thoroughly washed with methyl acetate and the combined filtrates were concentrated in vacuo. Silica gel column chromatography (AcOEt/cyclohexane 1:9) yielded compound $17(0.22 \mathrm{~g}, 85 \%)$ as a colorless oil. $[\alpha]_{\mathrm{D}}{ }^{21}-54\left(c 1.015, \mathrm{CHCl}_{3}\right)$; IR (neat) 1690, $1735 \mathrm{~cm}^{-1} ;{ }^{1} \mathrm{H} \mathrm{NMR}\left(\mathrm{CDCl}_{3}\right) \delta 1.15(\mathrm{~d}, J=6.25$ $\mathrm{Hz}, 3 \mathrm{H}), 1.36$ (s, 9H), 1.46-1.85 (m, 6H), 1.92 (s, 3H), 3.20-3.25 (m, 2H), 3.60-3.70 (m, 1H), 4.74-4.82 (m, 1H); ${ }^{13} \mathrm{C} \mathrm{NMR}\left(\mathrm{CDCl}_{3}\right) \delta 20.0,21.3,23.3,28.5,30.6,40.6,45.9,54.9,69.4,79.1$, 154.3, 170.4; Anal. Calcd for $\mathrm{C}_{14} \mathrm{H}_{25} \mathrm{NO}_{4}$ : C, 61.97; H, 9.29; N, 5.16. Found: C, 62.13; H, 9.36; N, 5.39.

(-)-Hygroline (13). To a suspension of $\mathrm{LiAlH}_{4}(310 \mathrm{mg}, 8.10 \mathrm{mmol})$ in dry THF $(10 \mathrm{~mL})$ was added dropwise a solution of compound $16(0.22 \mathrm{~g}, 0.81 \mathrm{mmol})$ in THF $(4 \mathrm{~mL})$. The reaction mixture was refluxed for $12 \mathrm{~h}$. After cooling to $0{ }^{\circ} \mathrm{C}$, water $(0.31 \mathrm{ml}), 15 \% \mathrm{NaOH}$ solution $(0.31$ $\mathrm{mL})$, water $(0.93 \mathrm{~mL})$ and anhydrous $\mathrm{K}_{2} \mathrm{CO}_{3}$ were successively added. The resulting reaction mixture was stirred at room temperature for $1 \mathrm{~h}$ and then filtered on a glass-frit. The residue was washed with THF. The solvent was carefully removed at room temperature under reduced pressure $(110 \mathrm{~mm} \mathrm{Hg})$ to yield the expected compound $(102 \mathrm{mg}, 87 \%)$. The spectroscopic data are in accordance with that of the literature. ${ }^{16,20,21}[\alpha]_{\mathrm{D}}{ }^{24}-50(c 1.28, \mathrm{MeOH})$. 


\section{References and Notes}

1. Negri, G.; Kascheres, C.; Kascheres, A. J. J. Heterocyclic Chem. 2004, 41, 461.

2. Michael, J. P.; de Koning, C. B.; Gravestock, D.; Hosken, G. D.; Howard, A. S.; Jungmann, C. M.; Krause, R. W. M.; Parsons, A. S.; Pelly, S. C; Stanbury, T. V. Pure Appl. Chem. 1999, 71, 979 .

3. (a) Ghirlando, R.; Howard, A. S.; Katz, R. B.; Michael, J. P. Tetrahedron 1984, 40, 2879.

(b) Michael, J. P.; Parsons, A. S. Tetrahedron 1996, 52, 2199. (c) Calvet-Vitale, S.; Vanucci-Bacqué, C.; Fargeau-Bellassoued, M.-C.; Lhommet, G. Tetrahedron, 2005, 61, 7774.

4. (a) Fréville, S.; Delbecq, P.; Thuy, V. M.; Petit, H.; Célérier, J.-P.; Lhommet, G. Tetrahedron Lett. 2001, 42, 4609. (b) Ma, D.; Zhu, W. Org. Lett. 2001, 3, 3927.

5. (a) Roth, M.; Dubs, P.; Götschi E.; Eschenmoser, A. Helv. Chem. Acta 1971, 54, 710. (b) Leete, E.; Bjorklund, J. A.; Couladis, M. M.; Kim, S. H. J. Am. Chem. Soc. 1991, 113, 9286. (c) Hernandez, A.; Rapoport, H. J. Org. Chem. 1994, 59, 1058. (d) Howard, M. H.; Sardina, F. J.; Rapoport, H. J. Org. Chem. 1990, 55, 2829. (e) Fuhrer, W., Rasetti, V.; Ribs, G. Helv. Chem. Acta 1985, 68, 1235. (f) David, O.; Blot, J.; Bellec, C.; Fargeau-Bellassoued, M.-C.; Haviari, G.; Célérier, J.-P.; Lhommet, G.; Gramain, J.-C.; Gardette, D. J. Org. Chem. 1999, 64,3122 .

6. (a) Harwood, L. M.; Tucker, T. T.; Angell, R.; Finch, H. Tetrahedron Lett. 1996, 37, 4217.

(b) Ségat, F.; Lingibé, O.; Graffe, B.; Sacquet, M.-C.; Lhommet, G. Heterocycles 1997, 45, 1451.

7. Delbecq, P. ; Célérier J.-P.; Lhommet, G. Tetrahedron Lett. 1990, 31, 4873.

8. David, O.; Calvet, S.; Chau, F.; Vanucci-Bacqué, C.; Fargeau-Bellassoued, M.-C.; Lhommet, G. J. Org. Chem. 2004, 69, 2888.

9. (a) Fodor, G. B.; Colasanti, B. The pyridine and piperidine alkaloids: chemistry and pharmacology; In Alkaloids: Chemical and biological perspectives; S. W. Pelletier, Ed. Pergamon: Oxford, 1985; Vol. 3, pp 1-90. (b) Southon, I. W.; Buckingham, J. In Dictionary of Alkaloids. Chapman and Hall: London, NewYork; 1989.

10. Buffet, M. F.; Dixon, D. J.; Ley, S. V.; Reynolds, D. J.; Storer, R. I. Org. Biomol. Chem. 2004, $2,1145$.

11. For 11b see: Dhondi, P. K.; Chisholm, J. D. Org. Lett. 2006, 8, 67.

12. The use of a mixture of $\mathrm{THF} / \mathrm{H}_{2} \mathrm{O}$ as the solvent instead of methanol led to degradation products.

13. Degnan, A. P.; Meyers, A. I. J. Org. Chem. 2000, 65, 3503.

14. Crystallographic data (excluding structure factors) for the structure of $\mathbf{6 b}$ have been deposited with the Cambridge Crystallographic Data Centre as supplementary publication number CCDC 626533. Copies of the data can be obtained, free of charge, on application to CCDC, 12 Union Road, Cambridge CB2 1EZ, UK (fax: +44-(0)1223-336033 or e-mail: deposit@ccdc.cam.ac.Uk). 
15. See for example: (a) Davies, S. G.; Haggitt, J. R.; Ichihara, O.; Kelly, R. J.; Leech, M. A.; Price Mortimer, A. J.; Roberts, P. M.; Smith, A. D. Org. Biomol. Chem. 2004, 2, 2630. (b) Davis, F. A.; Chao, B.; Fang, T.; Szewczyk, J. M. Org. Lett. 2000, 2, 1041. (c) Kozikowski, A. P.; Chen, Y.-Y. J. Org. Chem. 1981, 46, 5248.

16. Takahata, H.; Kubota M.; Momose, T. Tetrahedron: Asymmetry 1997, 16, 2801.

17. Bartoli, G.; Cimarelli, C.; Palmieri, G. J. J. Chem. Soc., Perkin Trans 1 1994, 537.

18. Bartoli, G.; Cupone, G.; Dalpozzo, R.; De Nino, A; Maiuolo, L.; Procopio, A.; Tagarelli, A. Tetrahedron Lett. 2002, 43, 7441.

19. Harris M. I. N. C.; Braga, A. C. H. J. Braz. Chem. Soc. 2004, 15, 971.

20. Louis C.; Hootelé, C. Tetrahedron: Asymmetry 1997, 8, 109.

21. Knight D. W.; Salter, R. Tetrahedron Lett. 1999, 40, 5915.

22. Lukes, R.; Kovár, J.; Kloubek, J.; Bláha, K. Coll. Czech. Chem. Comm. 1960, 483.

23. Palmieri G.; Cimarelli, C. Arkivoc 2006, (vi), 104. 